\title{
Magnetic interfaces as sources of coherent spin waves
}

\author{
V. D. Poimanov, ${ }^{1}$ A. N. Kuchko, ${ }^{2,3}$ and V. V. Kruglyak ${ }^{4, *}$ \\ ${ }^{1}$ Donetsk National University, 24 Universitetskaya Street, Donetsk 83001, Ukraine \\ ${ }^{2}$ Institute of Magnetism of NAS of Ukraine, $36 \mathrm{~b}$ Vernadskogo Avenue, Kiev 03142, Ukraine \\ ${ }^{3}$ Igor Sikorsky Kyiv Polytechnic Institute, 37 Prospect Peremohy, Kiev, 03056, Ukraine \\ ${ }^{4}$ University of Exeter, Stocker road, Exeter EX4 4QL, United Kingdom
}

(Received 24 July 2018; published 14 September 2018)

\begin{abstract}
We have developed a simple but general analytical theory that elucidates the mechanism of spin-wave generation from interfaces between ferromagnetic media pumped by a uniform microwave magnetic field. Our calculations show that, provided there is a finite coupling between the two media, the amplitude of the emitted spin waves depends linearly on the difference between their magnetic susceptibilities. The theory is successfully applied to interpret qualitatively three recent experimental studies in which such a spin-wave emission was observed. Furthermore, we describe how our approach can be extended to several more complicated spin-wave excitation schemes employing electric, elastic, and optical stimuli.
\end{abstract}

DOI: 10.1103/PhysRevB.98.104418

\section{INTRODUCTION}

A local perturbation of the magnetic order in magnetic materials may often propagate in the form of a special kind of waves: spin waves [1]. These are studied within the research field of magnonics [2,3], which derives its name from that of the magnon, a spin-wave quantum [4]. One of the greatest challenges in modern magnonics is the generation of spin waves with the shortest wavelength possible. Indeed, spin waves need to be generated before they can be studied or even used to transmit and manipulate data or electromagnetic signals within perceived magnonic devices [2]. Shorter wavelength would open both new avenues for magnonic research and opportunities for miniaturization of magnonic technology [5].

In the experiments to date, propagating coherent spin waves with the shortest wavelengths have been generated using spin-transfer torques (STTs) [6,7] and microwaves [8,9]. The latter scheme circumvents the challenge of confining the microwave magnetic field to the nanoscale by exploiting variants of the Schlömann mechanism of spin-wave excitation [10], which in turn can be interpreted in terms of Wigen's dynamic pinning [11]. This mechanism utilizes either natural $[9,10]$ or artificially created $[12,13]$ magnetic nonuniformities to break the translational symmetry and thereby to enable coupling between an essentially uniform microwave magnetic field and short-wavelength propagating spin waves. The Fanolike [14] mechanism of spin-wave excitation, demonstrated in Ref. [15] and then adopted in Ref. [8], may be regarded as a special case of the Schlömann scheme. However, both the existing experimental demonstrations and theoretical proposals have been limited to generation of coherent spin waves with wavelengths down to several tens of nanometers. The limit is ascribed to the dimensions of the relevant nonuniformity

\footnotetext{
*Corresponding author: v.v.kruglyak@exeter.ac.uk
}

acting as a spin-wave source, e.g., the STT device [6,7], the magnetic vortex core [9], or the lithographically defined magnonic transducer [15]. Hence, a question arises: What is the smallest wavelength of propagating coherent spin waves that could be controllably generated?

Here, we use a simple but general analytical theory to demonstrate that spin waves may be generated from magnetic interfaces pumped by the microwave magnetic field. Atomically flat magnetic interfaces have now become a common place in magnetic nanotechnology. Thus, we suggest that the interface-mediated spin-wave emission be the candidate mechanism for creation of ultimately small magnonic sources, limited only by the frequency dependence of the dynamic magnetic susceptibility of the adjacent media. We also use our theory to discuss qualitatively the recent experimental results on spin-wave generation and to argue that they are explained by the Schlömann mechanism [10].

The paper is organized as follows. In the next section (Sec. II), we present the main theoretical result of our study, i.e., the method that reduces the problem of spin-wave emission from a magnetic interface to inhomogeneous boundary conditions. The method is then discussed in Sect III: its general properties are discussed in Sec. III A, its use is illustrated using a simple problem as an example in Sec. III B; and several recent experimental results are discussed and interpreted using obtained insights in Sec. III C. Finally, Sec. IV is devoted to conclusions and outlook for further development of the theory in view of outstanding challenges.

\section{MODEL AND MAIN RESULTS}

Let us consider the interface between two semi-infinite magnetic media, $\mathrm{A}(z<-a / 2)$ and $\mathrm{B}(z>+a / 2)$, as shown in Fig. 1. Here, $a$ is the thickness of the spacer layer, which may be equal to the atomic lattice constant in the case of an atomically thin interface. The interface is parallel to the $x-y$ plane. The magnetization dynamics in the system are 


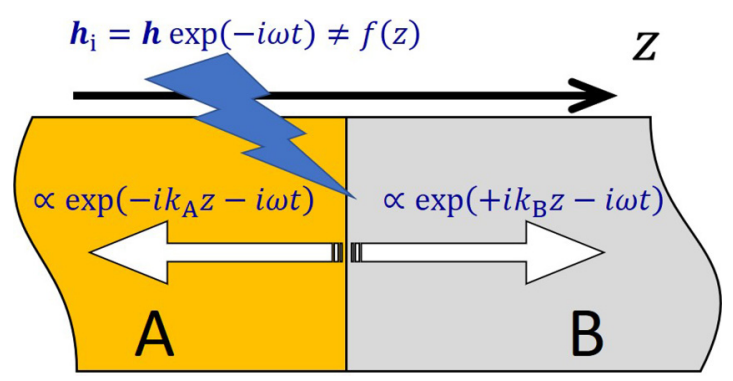

FIG. 1. The geometry of the problem is schematically shown. Two semi-infinite magnonic media $\mathrm{A}$ and $\mathrm{B}$ are irradiated by a uniform microwave magnetic field $\boldsymbol{h}_{\mathrm{i}}$, which leads to emission of spin waves from the interface into the media.

described by the Landau-Lifshitz equation

$$
\frac{\partial \boldsymbol{M}}{\partial t}=-\gamma\left[\boldsymbol{M} \times \boldsymbol{H}_{\mathrm{eff}}\right]+\frac{\alpha}{M}\left[\boldsymbol{M} \times \frac{\partial \boldsymbol{M}}{\partial t}\right],
$$

where $\boldsymbol{M}$ is the magnetization $\left(\boldsymbol{M}=\boldsymbol{M}_{\mathrm{A}}\right.$ for $z<-a / 2$ and $\boldsymbol{M}=\boldsymbol{M}_{\mathrm{B}}$ for $\left.z>+a / 2\right), M$ is the saturation magnetization $\left(M=M_{\mathrm{A}}\right.$ for $z<-a / 2$ and $M=M_{\mathrm{B}}$ for $\left.z>+a / 2\right), \gamma$ is the gyromagnetic ratio, $\alpha$ is the dimensionless (Gilbert) damping constant, and $t$ is the time. Both $\gamma$ and $\alpha$ are assumed to be constant in the entire sample, for the sake of brevity. The effective magnetic field $\boldsymbol{H}_{\text {eff }}$ is assumed to have form

$$
\boldsymbol{H}_{\mathrm{eff}}=\left\{\begin{array}{lc}
\boldsymbol{H}_{\mathrm{A}} & \left(z<-\frac{a}{2}\right), \\
\boldsymbol{H}_{\mathrm{B}} & \left(z>+\frac{a}{2}\right),
\end{array}\right.
$$

where

$$
\boldsymbol{H}_{\mathrm{A}(\mathrm{B})}=\frac{\partial}{\partial z}\left(\frac{2 A_{\mathrm{A}(\mathrm{B})}}{M_{\mathrm{A}(\mathrm{B})}^{2}} \frac{\partial \boldsymbol{M}_{\mathrm{A}(\mathrm{B})}}{\partial z}\right)+\boldsymbol{H}_{\mathrm{A}(\mathrm{B}), \mathrm{v}}+\boldsymbol{H}_{\mathrm{A}(\mathrm{B}), \mathrm{c}}+\boldsymbol{h}_{\mathrm{i}},
$$

$\boldsymbol{h}_{\mathrm{i}}$ is the incident microwave field, $A$ is the exchange constant $\left(A=A_{\mathrm{A}}\right.$ for $z<-a / 2$ and $A=A_{\mathrm{B}}$ for $\left.z>+a / 2\right)$, and indices "c" and " $\mathrm{v}$ " denote the interfacial coupling and volume contributions to the effective field, respectively. The incident microwave field is assumed to be effectively uniform on the length scales of the problem. The volume fields may be slowly varying functions of the coordinates. The interfacial coupling field is assumed here to have the following form:

$$
\begin{aligned}
\boldsymbol{H}_{\mathrm{A}, \mathrm{c}} & =\frac{2 A_{\mathrm{AB}}}{M_{\mathrm{A}} M_{\mathrm{B}}} \delta(z+a / 2) \boldsymbol{M}_{\mathrm{B}}(z+a), \\
\boldsymbol{H}_{\mathrm{B}, \mathrm{c}} & =\frac{2 A_{\mathrm{AB}}}{M_{\mathrm{A}} M_{\mathrm{B}}} \delta(z-a / 2) \boldsymbol{M}_{\mathrm{A}}(z-a),
\end{aligned}
$$

where $A_{\mathrm{AB}}$ is the interfacial coupling strength.

Assuming that the static distribution of the magnetization in the sample is known and given by $\boldsymbol{M}_{0}$, we can represent the magnetization and effective field as sums of their static values and small dynamic perturbations,

$$
\begin{aligned}
\boldsymbol{M} & =\boldsymbol{M}_{0}+\boldsymbol{m}(t), \quad \boldsymbol{H}_{\mathrm{eff}}=\boldsymbol{H}_{\mathrm{eff}, 0}+\boldsymbol{h}_{\mathrm{eff}}(t), \\
m & \ll M, \quad h_{\mathrm{eff}} \ll H_{\mathrm{eff}},
\end{aligned}
$$

and then linearize the Landau-Lifshitz equation in $\boldsymbol{m}$ and $\boldsymbol{h}_{\mathrm{eff}}$. As a result, we obtain

$$
\frac{\partial \boldsymbol{m}}{\partial t}=-\gamma\left[\boldsymbol{m} \times \boldsymbol{H}_{\mathrm{eff}, 0}\right]-\gamma\left[\boldsymbol{M}_{0} \times \boldsymbol{h}_{\mathrm{eff}}\right]+\frac{\alpha}{M}\left[\boldsymbol{M}_{0} \times \frac{\partial \boldsymbol{m}}{\partial t}\right] .
$$

By integrating the linearized Landau-Lifshitz equation without the damping term over infinitely small vicinities of the interface in each medium $[1,16,17]$, we obtain the following pair of boundary conditions for the dynamic magnetization:

$$
\begin{aligned}
& \frac{A_{\mathrm{AB}}}{M_{\mathrm{B}}}\left[\boldsymbol{m}_{\mathrm{A}} \times \boldsymbol{M}_{\mathrm{B}, 0}\right]+\frac{A_{\mathrm{AB}}}{M_{\mathrm{B}}}\left[\boldsymbol{M}_{\mathrm{A}, 0} \times \boldsymbol{m}_{\mathrm{B}}\right] \\
& -\frac{A_{\mathrm{A}}}{M_{\mathrm{A}}}\left[\boldsymbol{M}_{\mathrm{A}, 0} \times \frac{\partial \boldsymbol{m}_{\mathrm{A}}}{\partial z}\right]=0, \\
& \frac{A_{\mathrm{AB}}}{M_{\mathrm{A}}}\left[\boldsymbol{m}_{\mathrm{B}} \times \boldsymbol{M}_{\mathrm{A}, 0}\right]+\frac{A_{\mathrm{AB}}}{M_{\mathrm{A}}}\left[\boldsymbol{M}_{\mathrm{B}, 0} \times \boldsymbol{m}_{\mathrm{A}}\right] \\
& +\frac{A_{\mathrm{B}}}{M_{\mathrm{B}}}\left[\boldsymbol{M}_{\mathrm{B}, 0} \times \frac{\partial \boldsymbol{m}_{\mathrm{B}}}{\partial z}\right]=0 .
\end{aligned}
$$

Here, the function values are assumed to be taken at the respective interface boundaries in each medium rather than in the same point, e.g., $z=0[18,19]$.

We proceed to finding the response of our sample to the excitation by the uniform microwave magnetic field $\boldsymbol{h}_{\mathrm{i}}=$ $\boldsymbol{h} \exp (-i \omega t)$. Following the method from Refs. [20,21], we seek solutions in the form

$$
\boldsymbol{m}(t)=\boldsymbol{m}_{\mathrm{U}} \exp (-i \omega t)+\boldsymbol{\mu} \exp (-i \omega t)
$$

where $\boldsymbol{\mu}$ is the new unknown function and $\boldsymbol{m}_{\mathrm{U}}$ describes the linear response of the system to the incident uniform microwave magnetic field in the absence of the interfacial coupling, i.e., when $A_{\mathrm{AB}}$ is equal zero. Hence, $\boldsymbol{m}_{\mathrm{U}}$ satisfies in each medium the following inhomogeneous linear differential equations:

$$
\begin{aligned}
i \omega \boldsymbol{m}_{\mathrm{U}}= & \gamma\left[\boldsymbol{m}_{\mathrm{U}} \times \boldsymbol{H}_{\mathrm{v}, 0}\right]+\gamma\left[\boldsymbol{M}_{0} \times\left(\frac{\partial}{\partial z}\left(\frac{2 A}{M^{2}} \frac{\partial \boldsymbol{m}_{\mathrm{U}}}{\partial z}\right)\right.\right. \\
& \left.\left.+\boldsymbol{h}_{\mathrm{v}}\left(\boldsymbol{m}_{\mathrm{U}}\right)+\boldsymbol{h}\right)\right]+\frac{i \alpha \omega}{M}\left[\boldsymbol{M}_{0} \times \boldsymbol{m}_{\mathrm{U}}\right],
\end{aligned}
$$

where $\boldsymbol{H}_{\mathrm{v}, 0}$ and $\boldsymbol{h}_{\mathrm{v}}$ are the static and dynamic components of the volume contribution to the effective field $\left(h_{\mathrm{v}} \ll H_{\mathrm{v}, 0}\right)$, with the homogeneous boundary conditions

$$
\left[\boldsymbol{M}_{0} \times \frac{\partial \boldsymbol{m}_{\mathrm{U}}}{\partial z}\right]=0 .
$$

The solution can be written in a general form as

$$
\boldsymbol{m}_{\mathrm{A}(\mathrm{B}), \mathrm{U}}=\hat{\chi}_{\mathrm{A}(\mathrm{B})} \boldsymbol{h},
$$

where $\hat{\chi}_{\mathrm{A}(\mathrm{B})}$ are the susceptibility tensors of the media. We note that, since we have allowed the magnetic parameters of the two media to vary in space, both $\boldsymbol{m}_{\mathrm{A}(\mathrm{B}), \mathrm{U}}$ and $\hat{\chi}_{\mathrm{A}(\mathrm{B})}$ are also functions of the coordinates and should be treated as local functions [22]. This is not a problem, however, for the present calculation since we will only need their values at the interface boundaries.

The spin waves emitted from the interface are described by function $\boldsymbol{\mu}$, which is found from the homogeneous linear 
differential equation

$$
\begin{aligned}
i \omega \boldsymbol{\mu}= & \gamma\left[\boldsymbol{\mu} \times \boldsymbol{H}_{\mathrm{v}, 0}\right]+\gamma\left[\boldsymbol{M}_{0} \times\left(\frac{\partial}{\partial z}\left(\frac{2 A}{M^{2}} \frac{\partial \boldsymbol{\mu}}{\partial z}\right)+\boldsymbol{h}_{\mathrm{v}}(\boldsymbol{\mu})\right)\right] \\
& +\frac{i \alpha \omega}{M}\left[\boldsymbol{M}_{0} \times \boldsymbol{\mu}\right]
\end{aligned}
$$

with the inhomogeneous boundary conditions

$$
\begin{aligned}
& \frac{A_{\mathrm{AB}}}{M_{\mathrm{B}}} {\left[\boldsymbol{\mu}_{\mathrm{A}} \times \boldsymbol{M}_{\mathrm{B}, 0}\right]+\frac{A_{\mathrm{AB}}}{M_{\mathrm{B}}}\left[\boldsymbol{M}_{\mathrm{A}, 0} \times \boldsymbol{\mu}_{\mathrm{B}}\right] } \\
&-\frac{A_{\mathrm{A}}}{M_{\mathrm{A}}}\left[\boldsymbol{M}_{\mathrm{A}, 0} \times \frac{\partial \boldsymbol{\mu}_{\mathrm{A}}}{\partial z}\right] \\
&= \frac{A_{\mathrm{AB}}}{M_{\mathrm{B}}}\left[\boldsymbol{M}_{\mathrm{B}, 0} \times \hat{\chi}_{\mathrm{A}} \boldsymbol{h}\right]+\frac{A_{\mathrm{AB}}}{M_{\mathrm{B}}}\left[\hat{\chi}_{\mathrm{B}} \boldsymbol{h} \times \boldsymbol{M}_{\mathrm{A}, 0}\right], \\
& \frac{A_{\mathrm{AB}}}{M_{\mathrm{A}}}\left[\boldsymbol{\mu}_{\mathrm{B}} \times \boldsymbol{M}_{\mathrm{A}, 0}\right]+\frac{A_{\mathrm{AB}}}{M_{\mathrm{A}}}\left[\boldsymbol{M}_{\mathrm{B}, 0} \times \boldsymbol{\mu}_{\mathrm{A}}\right] \\
& \quad+\frac{A_{\mathrm{B}}}{M_{\mathrm{B}}}\left[\boldsymbol{M}_{\mathrm{B}, 0} \times \frac{\partial \boldsymbol{\mu}_{\mathrm{B}}}{\partial z}\right] \\
&=\frac{A_{\mathrm{AB}}}{M_{\mathrm{A}}}\left[\boldsymbol{M}_{\mathrm{A}, 0} \times \hat{\chi}_{\mathrm{B}} \boldsymbol{h}\right]+\frac{A_{\mathrm{AB}}}{M_{\mathrm{A}}}\left[\hat{\chi}_{\mathrm{A}} \boldsymbol{h} \times \boldsymbol{M}_{\mathrm{B}, 0}\right] .
\end{aligned}
$$

Adding and subtracting the two boundary conditions, we can also write them as

$$
\begin{aligned}
& \frac{2 A_{\mathrm{AB}}}{M_{\mathrm{A}} M_{\mathrm{B}}}\left[\boldsymbol{\mu}_{\mathrm{A}} \times \boldsymbol{M}_{\mathrm{B}, 0}\right]+\frac{2 A_{\mathrm{AB}}}{M_{\mathrm{A}} M_{\mathrm{B}}}\left[\boldsymbol{M}_{\mathrm{A}, 0} \times \boldsymbol{\mu}_{\mathrm{B}}\right] \\
& \quad-\frac{A_{\mathrm{A}}}{M_{\mathrm{A}}^{2}}\left[\boldsymbol{M}_{\mathrm{A}, 0} \times \frac{\partial \boldsymbol{\mu}_{\mathrm{A}}}{\partial z}\right]-\frac{A_{\mathrm{B}}}{M_{\mathrm{B}}^{2}}\left[\boldsymbol{M}_{\mathrm{B}, 0} \times \frac{\partial \boldsymbol{\mu}_{\mathrm{B}}}{\partial z}\right] \\
& =\frac{2 A_{\mathrm{AB}}}{M_{\mathrm{A}} M_{\mathrm{B}}}\left(\left[\boldsymbol{M}_{\mathrm{B}, 0} \times \hat{\chi}_{\mathrm{A}} \boldsymbol{h}\right]+\left[\hat{\chi}_{\mathrm{B}} \boldsymbol{h} \times \boldsymbol{M}_{\mathrm{A}, 0}\right]\right), \\
& \frac{A_{\mathrm{B}}}{M_{\mathrm{B}}^{2}}\left[\boldsymbol{M}_{\mathrm{B}, 0} \times \frac{\partial \boldsymbol{\mu}_{\mathrm{B}}}{\partial z}\right]=\frac{A_{\mathrm{A}}}{M_{\mathrm{A}}^{2}}\left[\boldsymbol{M}_{\mathrm{A}, 0} \times \frac{\partial \boldsymbol{\mu}_{\mathrm{A}}}{\partial z}\right] .
\end{aligned}
$$

In practice, one would use these boundary conditions to tailor two plane spin-wave solutions either propagating or decaying away from the interface on its either side. The inhomogeneity in the right-hand side of the first of Eq. (14) would then result in the two waves having nonzero amplitudes, i.e., in the emission of spin waves from the interface. Hence, as already mentioned earlier, the values of the static magnetizations $\boldsymbol{M}_{\mathrm{A}(\mathrm{B}), 0}$, spin-wave functions $\boldsymbol{\mu}_{\mathrm{A}(\mathrm{B})}$ and susceptibilities $\hat{\chi}_{\mathrm{A}(\mathrm{B})}$ are taken in the immediate vicinity of the interface boundaries. This means that the boundary conditions should be applicable to a range of problems beyond the exchange approximation assumed here. Only the physical nature and so the value of the coupling coefficient would need to be revised. This and other generalizations are discussed in greater detail in the rest of the paper.

\section{DISCUSSION}

\section{A. General properties of the inhomogeneous boundary conditions}

Let us begin by considering the general properties of the obtained boundary conditions (14). We observe that, if the coupling constant $A_{\mathrm{AB}}$ is equal zero, they become

$$
\begin{aligned}
& \frac{A_{\mathrm{B}}}{M_{\mathrm{B}}^{2}}\left[\boldsymbol{M}_{\mathrm{B}, 0} \times \frac{\partial \boldsymbol{\mu}_{\mathrm{B}}}{\partial z}\right]=-\frac{A_{\mathrm{A}}}{M_{\mathrm{A}}^{2}}\left[\boldsymbol{M}_{\mathrm{A}, 0} \times \frac{\partial \boldsymbol{\mu}_{\mathrm{A}}}{\partial z}\right], \\
& \frac{A_{\mathrm{B}}}{M_{\mathrm{B}}^{2}}\left[\boldsymbol{M}_{\mathrm{B}, 0} \times \frac{\partial \boldsymbol{\mu}_{\mathrm{B}}}{\partial z}\right]=\frac{A_{\mathrm{A}}}{M_{\mathrm{A}}^{2}}\left[\boldsymbol{M}_{\mathrm{A}, 0} \times \frac{\partial \boldsymbol{\mu}_{\mathrm{A}}}{\partial z}\right],
\end{aligned}
$$

which are equivalent to the boundary conditions (10) for $\boldsymbol{m}_{\mathrm{U}}$. However, the differential equation for $\boldsymbol{\mu}_{\mathrm{A}(\mathrm{B})}$ is homogeneous. This means that, for each interface boundary independently, the complex amplitudes of the incident and outgoing waves must be equal, and so no spin-wave emission is possible. This is also obvious, of course, from the fact that neither the differential equation (9) nor the boundary conditions (10) have any source terms in them. For the same reason, no spin-wave emission is possible if the source term $\boldsymbol{\Theta}=$ $\frac{2 A_{\mathrm{AB}}}{M_{\mathrm{A}} M_{\mathrm{B}}}\left(\left[\boldsymbol{M}_{\mathrm{B}, 0} \times \hat{\chi}_{\mathrm{A}} \boldsymbol{h}\right]+\left[\hat{\chi}_{\mathrm{B}} \boldsymbol{h} \times \boldsymbol{M}_{\mathrm{A}, 0}\right]\right)$ in Eq. (14) becomes equal to zero for any other reason. Depending on the problem, the local microwave susceptibility tensors $\hat{\chi}_{\mathrm{A}(\mathrm{B})}$ in the vicinity of the interface can be calculated analytically [23] or from micromagnetic simulations [22,24]. The most efficient emission is expected when the source term is strongest. This is achieved when either one or the other of the two media is driven in or close to resonance. If the incident microwave magnetic field excites both media with comparable strength, the relative phase of the uniform precessions $\boldsymbol{m}_{\mathrm{A}(\mathrm{B}), \mathrm{U}}$ induced in the two media needs to be considered.

In the case of a very strong coupling, we obtain

$$
\begin{aligned}
& {\left[\boldsymbol{\mu}_{\mathrm{A}} \times \boldsymbol{M}_{\mathrm{B}, 0}\right]+\left[\boldsymbol{M}_{\mathrm{A}, 0} \times \boldsymbol{\mu}_{\mathrm{B}}\right]} \\
& \quad=\left[\boldsymbol{M}_{\mathrm{B}, 0} \times \hat{\chi}_{\mathrm{A}} \boldsymbol{h}\right]+\left[\hat{\chi}_{\mathrm{B}} \boldsymbol{h} \times \boldsymbol{M}_{\mathrm{A}, 0}\right], \\
& \frac{A_{\mathrm{B}}}{M_{\mathrm{B}}^{2}}\left[\boldsymbol{M}_{\mathrm{B}, 0} \times \frac{\partial \boldsymbol{\mu}_{\mathrm{B}}}{\partial z}\right]=\frac{A_{\mathrm{A}}}{\boldsymbol{M}_{\mathrm{A}}^{2}}\left[\boldsymbol{M}_{\mathrm{A}, 0} \times \frac{\partial \boldsymbol{\mu}_{\mathrm{A}}}{\partial z}\right],
\end{aligned}
$$

i.e. a pair of boundary conditions that are independent of $A_{\mathrm{AB}}$. In other words, although the coupling between the two media is a prerequisite for the observation of the spin-wave emission, once the two magnonic media are coupled, the exact strength of the coupling becomes a secondary factor for the emission process.

\section{B. Example of a full solution of a simple problem}

Now let us consider the case when the static magnetizations near the interface in the two media are parallel, i.e., $\boldsymbol{M}_{\mathrm{A}, 0} \| \boldsymbol{M}_{\mathrm{B}, 0}$. The two media have axially symmetric properties, e.g., they have uniaxial anisotropies with different strengths $K_{\mathrm{A}(\mathrm{B})}$ but with the parallel axes of the easy magnetization (e.g., $\hat{z}$ ) along which the media are magnetized by a magnetic field with strength $H$. In this simple model, the spin waves in both media are circularly polarized and can be described by scalar functions

$$
\mu_{\mathrm{A}(\mathrm{B})} \propto \exp \left( \pm i k_{\mathrm{A}(\mathrm{B})} z\right),
$$

where only propagation along the $\hat{z}$ axis is considered. Their complex wave numbers are

$$
k_{\mathrm{A}(\mathrm{B})}=\sqrt{\frac{M_{\mathrm{A}(\mathrm{B})}}{2 A_{\mathrm{A}(\mathrm{B})}}\left(\omega-\omega_{0, \mathrm{~A}(\mathrm{~B})}+i \alpha \omega\right)},
$$


where $\omega_{0, \mathrm{~A}(\mathrm{~B})}=\gamma\left(H+\frac{2 K_{\mathrm{A}(\mathrm{B})}}{M_{\mathrm{A}(\mathrm{B})}}\right)$ are the uniform ferromagnetic resonance frequencies in the semi-infinite media.

The boundary conditions (14) for the emitted spin waves are then simplified to the following scalar form:

$$
\begin{aligned}
\frac{\mu_{\mathrm{B}}}{M_{\mathrm{B}}}-\frac{\mu_{\mathrm{A}}}{M_{\mathrm{A}}} & -\frac{A_{\mathrm{A}}}{2 A_{\mathrm{AB}} M_{\mathrm{A}}} \frac{\partial \mu_{\mathrm{A}}}{\partial z}-\frac{A_{\mathrm{B}}}{2 A_{\mathrm{AB}} M_{\mathrm{B}}} \frac{\partial \mu_{\mathrm{B}}}{\partial z} \\
& =\left(\frac{\chi_{\mathrm{A}}}{M_{\mathrm{A}}}-\frac{\chi_{\mathrm{B}}}{M_{\mathrm{B}}}\right) h \\
\frac{A_{\mathrm{B}}}{M_{\mathrm{B}}} \frac{\partial \mu_{\mathrm{B}}}{\partial z} & =\frac{A_{\mathrm{A}}}{M_{\mathrm{A}}} \frac{\partial \mu_{\mathrm{A}}}{\partial z},
\end{aligned}
$$

where $h$ is now the amplitude of the incident circularly polarized microwave magnetic field and the scalar microwave susceptibilities of the media are

$$
\chi_{\mathrm{A}(\mathrm{B})}=-\frac{M_{\mathrm{A}(\mathrm{B})}^{2}}{2 A_{\mathrm{A}(\mathrm{B})} k_{\mathrm{A}(\mathrm{B})}^{2}}=-\frac{\gamma M_{\mathrm{A}(\mathrm{B})}}{\omega-\omega_{0, \mathrm{~A}(\mathrm{~B})}+i \alpha \omega} .
$$

Considering only the waves propagating away from the interface, i.e., $\mu_{\mathrm{A}} \propto \exp \left(-i k_{\mathrm{A}} z\right)$ and $\mu_{\mathrm{B}} \propto \exp \left(+i k_{\mathrm{B}} z\right)$, and neglecting the interface thickness compared to the wavelength of the spin waves [17], we obtain

$$
\begin{aligned}
\frac{C_{\mathrm{B}}}{M_{\mathrm{B}}}-\frac{C_{\mathrm{A}}}{M_{\mathrm{A}}}+i \frac{k_{\mathrm{A}} A_{\mathrm{A}} C_{\mathrm{A}}}{2 A_{\mathrm{AB}} M_{\mathrm{A}}}-i \frac{k_{\mathrm{B}} A_{\mathrm{B}} C_{\mathrm{B}}}{2 A_{\mathrm{AB}} M_{\mathrm{B}}} & =\left(\frac{\chi_{\mathrm{A}}}{M_{\mathrm{A}}}-\frac{\chi_{\mathrm{B}}}{M_{\mathrm{B}}}\right) h, \\
\frac{k_{\mathrm{B}} A_{\mathrm{B}} C_{\mathrm{B}}}{M_{\mathrm{B}}} & =-\frac{k_{\mathrm{A}} A_{\mathrm{A}} C_{\mathrm{A}}}{M_{\mathrm{A}}},
\end{aligned}
$$

where $C_{\mathrm{A}(\mathrm{B})}$ are the complex amplitudes of the spin waves in media $\mathrm{A}$ and $\mathrm{B}$, respectively. The second of the equations requires the waves emitted on the opposite sides of the interface to have opposite phases. The full solutions of this system of equations are

$$
\begin{aligned}
C_{\mathrm{A}} & =\frac{k_{\mathrm{B}} A_{\mathrm{B}} A_{\mathrm{AB}} M_{\mathrm{A}}\left(\frac{\chi_{\mathrm{B}}}{M_{\mathrm{B}}}-\frac{\chi_{\mathrm{A}}}{M_{\mathrm{A}}}\right)}{\left(k_{\mathrm{A}} A_{\mathrm{A}}+k_{\mathrm{B}} A_{\mathrm{B}}\right) A_{\mathrm{AB}}-i k_{\mathrm{A}} A_{\mathrm{A}} k_{\mathrm{B}} A_{\mathrm{B}}} h, \\
C_{\mathrm{B}} & =\frac{k_{\mathrm{A}} A_{\mathrm{A}} A_{\mathrm{AB}} M_{\mathrm{B}}\left(\frac{\chi_{\mathrm{A}}}{M_{\mathrm{A}}}-\frac{\chi_{\mathrm{B}}}{M_{\mathrm{B}}}\right)}{\left(k_{\mathrm{A}} A_{\mathrm{A}}+k_{\mathrm{B}} A_{\mathrm{B}}\right) A_{\mathrm{AB}}-i k_{\mathrm{A}} A_{\mathrm{A}} k_{\mathrm{B}} A_{\mathrm{B}}} h .
\end{aligned}
$$

Thus, the uniform microwave magnetic field $\boldsymbol{h}_{\mathrm{i}}=$ $\boldsymbol{h} \exp (-i \omega t)$ incident on the interface $z=0$ between two semi-infinite ferromagnetic media A and B leads to emission from the interface of two propagating spin waves,

$$
\begin{aligned}
\boldsymbol{\mu}_{\mathrm{A}} e^{-i \omega t} & =\boldsymbol{h} \frac{k_{\mathrm{B}} A_{\mathrm{B}} A_{\mathrm{AB}} M_{\mathrm{A}}\left(\frac{\chi_{\mathrm{B}}}{M_{\mathrm{B}}}-\frac{\chi_{\mathrm{A}}}{M_{\mathrm{A}}}\right)}{\left(k_{\mathrm{A}} A_{\mathrm{A}}+k_{\mathrm{B}} A_{\mathrm{B}}\right) A_{\mathrm{AB}}-i k_{\mathrm{A}} A_{\mathrm{A}} k_{\mathrm{B}} A_{\mathrm{B}}} e^{-i k_{\mathrm{A}} z-i \omega t} \\
\text { for } z & <-a / 2, \\
\boldsymbol{\mu}_{\mathrm{B}} e^{-i \omega t} & =\boldsymbol{h} \frac{k_{\mathrm{A}} A_{\mathrm{A}} A_{\mathrm{AB}} M_{\mathrm{B}}\left(\frac{\chi_{\mathrm{A}}}{M_{\mathrm{A}}}-\frac{\chi_{\mathrm{B}}}{M_{\mathrm{B}}}\right)}{\left(k_{\mathrm{A}} A_{\mathrm{A}}+k_{\mathrm{B}} A_{\mathrm{B}}\right) A_{\mathrm{AB}}-i k_{\mathrm{A}} A_{\mathrm{A}} k_{\mathrm{B}} A_{\mathrm{B}}} e^{+i k_{\mathrm{B}} z-i \omega t} \\
\text { for } z & >+a / 2 .
\end{aligned}
$$

Equations (22) confirm our earlier observations that no emission is possible when the two media are either uncoupled (i.e., $A_{\mathrm{AB}}=0$ ) or excited equally (in terms of both amplitude and phase) near the interface (i.e., $\frac{\chi_{\mathrm{A}}}{M_{\mathrm{A}}}=\frac{\chi_{\mathrm{B}}}{M_{\mathrm{B}}}$ ), e.g., at a particular frequency. When one of the two media (e.g., medium A) is driven close to its resonance and the other (medium B) is relatively far from its resonance, we can approximately assume that $\frac{\chi_{\mathrm{A}}}{M_{\mathrm{A}}} \gg \frac{\chi_{\mathrm{B}}}{M_{\mathrm{B}}}, k_{\mathrm{B}} \gg k_{\mathrm{A}}$. This leads to

$$
\begin{aligned}
& C_{\mathrm{A}} \cong-\chi_{\mathrm{A}} h, \\
& C_{\mathrm{B}} \cong \frac{k_{\mathrm{A}} A_{\mathrm{A}} M_{\mathrm{B}}}{k_{\mathrm{B}} A_{\mathrm{B}} M_{\mathrm{A}}} \chi_{\mathrm{A}} h .
\end{aligned}
$$

The total amplitude of precession near the interface in medium $\mathrm{A}$ is then $m_{\mathrm{A}, \mathrm{U}}+C_{\mathrm{A}} \cong 0$, which corresponds to the dynamic pinning of the magnetization discovered by Wigen et al. [11]. Combining Eqs. (20) and (24), we see that the amplitude of spin waves emitted into medium B remains finite, and is

$$
C_{\mathrm{B}} \cong-\frac{M_{\mathrm{A}} M_{\mathrm{B}}}{2 k_{\mathrm{A}} k_{\mathrm{B}} A_{\mathrm{B}}} h,
$$

which is in fact quite large since $k_{\mathrm{A}}$ is small.

When the coupling is weak, i.e., when $A_{\mathrm{AB}}$ can be considered as a small parameter, Eqs. (22) predict a linear dependence of the amplitudes of the emitted spin waves on the coupling strength:

$$
\begin{gathered}
C_{\mathrm{A}} \approx i \frac{A_{\mathrm{AB}} M_{\mathrm{A}}\left(\frac{\chi_{\mathrm{B}}}{M_{\mathrm{B}}}-\frac{\chi_{\mathrm{A}}}{M_{\mathrm{A}}}\right)}{k_{\mathrm{A}} A_{\mathrm{A}}} \text { and } \\
C_{\mathrm{B}} \approx i \frac{A_{\mathrm{AB}} M_{\mathrm{B}}\left(\frac{\chi_{\mathrm{A}}}{M_{\mathrm{A}}}-\frac{\chi_{\mathrm{B}}}{M_{\mathrm{B}}}\right)}{k_{\mathrm{B}} A_{\mathrm{B}}} h .
\end{gathered}
$$

When the coupling is strong, i.e., when $A_{\mathrm{AB}}$ can be considered as a large parameter, Eqs. (22) predict that the dependence of the amplitudes of the emitted spin waves on the coupling strength saturates, so that the amplitudes approach asymptotically constant values:

$$
\begin{aligned}
& C_{\mathrm{A}} \approx \frac{k_{\mathrm{B}} A_{\mathrm{B}} M_{\mathrm{A}}\left(\frac{\chi_{\mathrm{B}}}{M_{\mathrm{B}}}-\frac{\chi_{\mathrm{A}}}{M_{\mathrm{A}}}\right)}{k_{\mathrm{A}} A_{\mathrm{A}}+k_{\mathrm{B}} A_{\mathrm{B}}} \text { and } \\
& C_{\mathrm{B}} \approx \frac{k_{\mathrm{A}} A_{\mathrm{A}} M_{\mathrm{B}}\left(\frac{\chi_{\mathrm{A}}}{M_{\mathrm{A}}}-\frac{\chi_{\mathrm{B}}}{M_{\mathrm{B}}}\right)}{k_{\mathrm{A}} A_{\mathrm{A}}+k_{\mathrm{B}} A_{\mathrm{B}}} h .
\end{aligned}
$$

Now, we consider the frequency dependence of the amplitudes of the emitted spin waves when the driving frequency is much greater than the ferromagnetic resonance frequencies of the media. Combining Eqs. (18) and (20) with Eqs. (26) and (27), we find that the amplitudes decrease as $\propto \omega^{-2}$ when the coupling is strong and $\propto \omega^{-5 / 2}$ when it is weak.

\section{Application to interpretation of recent experimental results}

We have identified three recent experimental studies [2527] to which the theory developed here could be applied. We discuss qualitatively the physics underpinning the observations in each case, making links to how our approach could be modified to account for various experimental situations.

Au et al. used time-resolved scanning Kerr microscopy (TRSKM) to study the magnetic response of a continuous junction between a semi-infinite film and a semi-infinite stripe [25]. The structure had micrometer-scale dimensions and was patterned from a rather thick film of permalloy. Hence, the spin waves observed by Au et al. to be emitted from the junction were of a purely magnetostatic nature, more specifically magnetostatic surface spin waves (MSSWs) [28]. From a glance, this seems to contradict the exchange approximation employed here. However, we note that the theory of MSSWs 
is based on a second-order differential equation, the Walker equation [29]. Hence, the same method as that presented here could be applied to quantify the emission of the MSSWs from the interface between the semi-infinite film and stripe, with the complication of needing to stitch the solutions for the magnetostatic potential at the interface not only within but also outside the magnetic material. As far as the wave emission is concerned, however, we expect the relevant physics to remain the same as here. The continuity of the magnetostatic potential and its derivatives at the interface ensures coupling between the waves in the two media. So, the only major factor expected to affect the MSSW emission should therefore be the difference of the microwave magnetic susceptibilities at the interface, or rather their gradient. Indeed, the variation of the magnetic susceptibility in Ref. [25] resulted from the shape anisotropy. The latter originates from the internal magnetic field, which may only have discontinuities at discontinuities of the magnetization. At the same time, we showed in Ref. [17] that interfaces of finite thickness could be described using boundary conditions of Barnaś-Mills form [18,19], which are fully compatible with the method described here.

Hämäläinen $e t$ al. observed emission of dipole-exchange spin waves in a thin-film sample with a periodically modulated orientation of the uniaxial anisotropy axis [26]. Their micromagnetic simulations were used to show that the spinwave emission could also be observed from a single region of increased effective magnetic field and from an isolated interface. Our theory lends a qualitative explanation to the observations of Hämäläinen et al. Neglecting the ellipticity of precession in the system studied in Ref. [26], the amplitudes of spin waves emitted from an isolated interface between media with different directions of the easy magnetization axes can be expected to be approximately given by

$$
\begin{aligned}
C_{\mathrm{A}} & =\frac{k_{\mathrm{B}}\left(\chi_{\mathrm{B}}-\chi_{\mathrm{A}}\right)}{k_{\mathrm{A}}+k_{\mathrm{B}}} h, \\
C_{\mathrm{B}} & =\frac{k_{\mathrm{A}}\left(\chi_{\mathrm{A}}-\chi_{\mathrm{B}}\right)}{k_{\mathrm{A}}+k_{\mathrm{B}}} h,
\end{aligned}
$$

where $k_{\mathrm{A}(\mathrm{B})}$ and $\chi_{\mathrm{A}(\mathrm{B})}$ include relevant magnetostatic contributions. In particular, this shows that the spin-wave emission occurs into both media with equal amplitudes and opposite phases, as we indeed observe in Fig. 6 from Ref. [26]. Again, the major contribution to the emission mechanism is the difference between the susceptibilities of the two media. A quantitative theory of the observations from Ref. [26], including the case of a periodic structure, is a topic of our ongoing investigations and will be presented elsewhere.

Klinger et al. observed formation of nonuniform standing spin waves in a film of yttrium-iron-garnet (YIG) covered by a layer of cobalt, either in a direct contact or separated by a nonmagnetic spacer layer [27]. Again, the observations of Klinger et al. fall squarely within both the picture of Wigen's dynamic pinning [11] and Schlömann's spin-wave excitation mechanism [10]. The calculations here expose the decisive role of the difference between the resonance frequencies in and therefore the microwave susceptibilities of the cobalt and YIG films. This conclusion is echoed by the observations of
Qin et al. based on micromagnetic simulations for similar structures [30]. At the same time, the exact nature of the interfacial coupling between cobalt and YIG is a secondary consideration, as far as the mechanism of the spin-wave emission is concerned. Again, the problems from Refs. [27,30] seem to allow for a full analytical solution, which is, however, a topic of a separate calculation. As an aside, we note that the dynamic pinning [11] like that observed in Ref. [27] can be used to design structures with increased magnetic permeability values at elevated frequencies $[31,32]$.

\section{CONCLUSIONS AND OUTLOOK}

In conclusion, we have developed a simple analytical theory of spin-wave emission from magnetic interfaces. The theory has been applied to interpret qualitatively three recent experimental studies in which spin-wave emission was observed from interfaces in magnetic structures. The major conclusion of the study is that the amplitude of emitted spin waves scales linearly with the difference between the magnetic susceptibilities of the adjacent magnonic media. At the same time, the dependence of the emitted spin-wave amplitude on the interlayer coupling strength is linear when the coupling is weak, and then saturates as the coupling strength increases, becoming constant in the strong coupling regime. Since the interfaces may be made virtually infinitely thin, the emitted spin waves may also have an arbitrary small wavelength. However, their amplitude is still limited by the frequency dependence of the magnetic susceptibility and in turn the values of the resonance frequencies of the adjacent media. Hence, a question arises as to how the resonance frequency of one of the two media could be increased. Possible strategies include the use of confined exchange spin waves [31,32], hexaferrites [8,33], or even antiferromagnets [34] and weak ferromagnets [35]. In particular, the use of antiferromagnets is capable of pushing the excitation frequencies to the $\mathrm{THz}$ domain but will require one to reconsider the choice of stimuli employed to excite the sample. Indeed, in this paper, we have only considered the process of spin-wave emission in response to excitation by an incident microwave magnetic field. The latter could be replaced by a field of another nature, e.g., an electric field [36-39], a strain field [40,41] or an effective magnetic field due to ultrashort optical pulses $[34,35,42,43]$. Both the theory and physics it describes would remain very similar, with the major difference being that the susceptibility $\hat{\chi}_{\mathrm{A}(\mathrm{B})}$ would need to be replaced by a quantity describing the response of the media to the relevant stimulus. Systematic theoretical and experimental realizations of the strategy presented in this paper to these and many other situations are topics for future research.

\section{ACKNOWLEDGMENTS}

The research leading to these results has received funding from the Engineering and Physical Sciences Research Council of the United Kingdom (Project No. EP/L019876/1) and from the European Union's Horizon 2020 research and innovation program under Marie Skłodowska-Curie Grant Agreement No. 644348 (MagIC). 
[1] A. I. Akhiezer, V. G. Bar'yakhtar, and S. V. Peletminskii, Spin Waves (North-Holland, Amsterdam, 1968).

[2] V. V. Kruglyak, S. O. Demokritov, and D. Grundler, Magnonics, J. Phys. D 43, 264001 (2010).

[3] S. A. Nikitov, D. V. Kalyabin, I. V. Lisenkov, A. Slavin, Y. N. Barabanenkov, S. A. Osokin, A. V. Sadovnikov, E. N. Beginin, M. A. Morozova, Y. P. Sharaevsky et al., Magnonics: A new research area in spintronics and spin wave electronics, Phys. Usp. 58, 1002 (2015).

[4] F. Bloch, Zur Theorie des Ferromagnetismus, Z. Phys. 61, 206 (1930).

[5] D. Grundler, Nanomagnonics, J. Phys. D 49, 391002 (2016).

[6] S. Urazhdin, V. E. Demidov, H. Ulrichs, T. Kendziorczyk, T. Kuhn, J. Leuthold, G. Wilde, and S. O. Demokritov, Nanomagnonic devices based on the spin-transfer torque, Nat. Nanotechnol. 9, 509 (2014).

[7] A. Houshang, E. Iacocca, P. Durrenfeld, S. R. Sani, J. Akerman, and R. K. Dumas, Spin-wave-beam driven synchronization of nanocontact spin-torque oscillators, Nat. Nanotechnol. 11, 280 (2016).

[8] H. M. Yu, O. D. Kelly, V. Cros, R. Bernard, P. Bortolotti, A. Anane, F. Brandl, F. Heimbach, and D. Grundler, Approaching soft X-ray wavelengths in nanomagnet-based microwave technology, Nat. Commun. 7, 11255 (2016).

[9] S. Wintz, V. Tiberkevich, M. Weigand, J. Raabe, J. Lindner, A. Erbe, A. Slavin, and J. Fassbender, Magnetic vortex cores as tunable spin-wave emitters, Nat. Nanotechnol. 11, 948 (2016).

[10] E. Schlömann, Generation of spin waves in nonuniform magnetic field. I. Conversion of electromagnetic power into spinwave power and vice versa, J. Appl. Phys. 35, 159 (1964).

[11] P. E. Wigen, C. F. Kooi, M. R. Shanabarger, and T. D. Rossing, Dynamic Pinning in Thin-Film Spin-Wave Resonance, Phys. Rev. Lett. 9, 206 (1962).

[12] P. E. Zilberman, A. G. Temiryazev, and M. P. Tikhomirova, Excitation and dispersion of exchange spin waves in iron-yttrium garnet films, Zh. Eksp. Teor. Fiz. 108, 281 (1995).

[13] C. S. Davies and V. V. Kruglyak, Generation of propagating spin waves from edges of magnetic nanostructures pumped by uniform microwave magnetic field, IEEE Trans. Magn. 52, 2300504 (2016).

[14] U. Fano, Effects of configuration interaction on intensities and phase shifts, Phys. Rev. 124, 1866 (1961).

[15] Y. Au, E. Ahmad, O. Dmytriiev, M. Dvornik, T. Davison, and V. V. Kruglyak, Resonant microwave-to-spin-wave transducer, Appl. Phys. Lett. 100, 182404 (2012).

[16] G. T. Rado and J. R. Weertman, Spin-wave resonance in a ferromagnetic metal, J. Phys. Chem. Solids 11, 315 (1959).

[17] V. V. Kruglyak, O. Y. Gorobets, Y. I. Gorobets, and A. N. Kuchko, Magnetization boundary conditions at a ferromagnetic interface of finite thickness, J. Phys.: Condens. Matter 26, 406001 (2014).

[18] J. Barnaś, On the Hoffmann boundary conditions at the interface between two ferromagnets, J. Magn. Magn. Mater. 102, 319 (1991).

[19] D. L. Mills, Spin waves in ultrathin exchange-coupled ferromagnetic multilayers: The boundary conditions at the interface, Phys. Rev. B 45, 13100 (1992).
[20] Y. I. Gorobets, A. N. Kuchko, and S. V. Vasil'yev, Excitation of modulated spin waves by model one-dimension anisotropy defect, Fiz. Met. Metalloved. 85, 40 (1998).

[21] N. J. Whitehead, S. A. R. Horsley, T. G. Philbin, A. N. Kuchko, and V. V. Kruglyak, Theory of linear spin wave emission from a Bloch domain wall, Phys. Rev. B 96, 064415 (2017).

[22] C. S. Davies, V. D. Poimanov, and V. V. Kruglyak, Mapping the magnonic landscape in patterned magnetic structures, Phys. Rev. B 96, 094430 (2017).

[23] A. N. Marchenko and V. N. Krivoruchko, Magnetic structure and resonance properties of a hexagonal lattice of antidots, Low. Temp. Phys. 38, 157 (2012).

[24] F. B. Mushenok, R. Dost, C. S. Davies, D. A. Allwood, B. Inkson, G. Hrkac, and V. V. Kruglyak, Broadband conversion of microwaves into propagating spin waves in patterned magnetic structures, Appl. Phys. Lett. 111, 042404 (2017).

[25] Y. Au, T. Davison, E. Ahmad, P. S. Keatley, R. J. Hicken, and V. V. Kruglyak, Excitation of propagating spin waves with global uniform microwave fields, Appl. Phys. Lett. 98, 122506 (2011).

[26] S. J. Hämäläinen, F. Brandl, K. J. A. Franke, D. Grundler, and S. van Dijken, Tunable Short-Wavelength Spin-Wave Emission and Confinement in Anisotropy-Modulated Multiferroic Heterostructures, Phys. Rev. Appl. 8, 014020 (2017).

[27] S. Klingler, V. Amin, S. Geprägs, K. Ganzhorn, H. Maier-Flaig, M. Althammer, H. Huebl, R. Gross, R. D. McMichael, M. D. Stiles, S. T. B. Goennenwein, and M. Weiler, Spin-Torque Excitation of Perpendicular Standing Spin Waves in Coupled YIG/Co Heterostructures, Phys. Rev. Lett. 120, 127201 (2018).

[28] J. R. Eshbach and R. W. Damon, Surface magnetostatic modes and surface spin waves, Phys. Rev. 118, 1208 (1960).

[29] L. R. Walker, Magnetostatic modes in ferromagnetic resonance, Phys. Rev. 105, 390 (1957).

[30] H. Qin, S. J. Hämäläinen, and S. van Dijken, Exchange-torqueinduced excitation of perpendicular standing spin waves in nanometer-thick YIG films, Sci. Rep. 8, 5755 (2018).

[31] R. V. Mikhaylovskiy, E. Hendry, and V. V. Kruglyak, Negative permeability due to exchange spin-wave resonances in thin magnetic films with surface pinning, Phys. Rev. B 82, 195446 (2010).

[32] M. Mruczkiewicz, M. Krawczyk, R. V. Mikhaylovskiy, and V. V. Kruglyak, Towards high-frequency negative permeability using magnonic crystals in metamaterial design, Phys. Rev. B 86, 024425 (2012).

[33] S. G. Appleton, Field dependence of FMR in ferrites and implications for microwave absorber design, Proc. SPIE 2448, 22 (1995).

[34] V. V. Kruglyak and R. J. Hicken, Magnonics: Experiment to Prove the concept, J. Magn. Magn. Mater. 306, 191 (2006).

[35] R. V. Mikhaylovskiy, E. Hendry, A. Secchi, J. H. Mentink, M. Eckstein, A. Wu, R. V. Pisarev, V. V. Kruglyak, M. I. Katsnelson, T. Rasing, and A. V. Kimel, Ultrafast optical modification of exchange interactions in iron oxides, Nat. Commun. 6, 8190 (2015).

[36] L. Chaix, S. de Brion, S. Petit, R. Ballou, L.-P. Regnault, J. Ollivier, J. B. Brubach, P. Roy, J. Debray, P. Lejay, A. Cano, E. Ressouche, and V. Simonet, Magneto- to Electroactive Transmutation of Spin Waves in $\mathrm{ErMnO}_{3}$, Phys. Rev. Lett. 112, 137201 (2014). 
[37] H.-B. Chen, Y.-Q. Li, and J. Berakdar, Electric-field control of electromagnon propagation and spin-wave injection in a spiral multiferroic/ferromagnet composite, J. Appl. Phys. 117, 043910 (2015).

[38] W. Chen and M. Sigrist, Dissipationless Multiferroic Magnonics, Phys. Rev. Lett. 114, 157203 (2015).

[39] S. Baierl, M. Hohenleutner, T. Kampfrath, A. K. Zvezdin, A. V. Kimel, R. Huber, and R. V. Mikhaylovskiy, Nonlinear spin control by terahertz-driven anisotropy fields, Nat. Photon. 10, 715 (2016).

[40] A. Kamra, H. Keshtgar, P. Yan, and G. E. W. Bauer, Coherent elastic excitation of spin waves, Phys. Rev. B 91, 104409 (2015).
[41] P. Graczyk, J. Klos, and M. Krawczyk, Broadband magnetoelastic coupling in magnonic-phononic crystals for high-frequency nanoscale spin-wave generation, Phys. Rev. B 95, 104425 (2017).

[42] A. V. Kimel, A. Kirilyuk, P. A. Usachev, R. V. Pisarev, A. M. Balbashov, and T. Rasing, Ultrafast non-thermal control of magnetization by instantaneous photomagnetic pulses, Nature (London) 435, 655 (2005).

[43] R. V. Mikhaylovskiy, T. J. Huisman, R. V. Pisarev, T. Rasing, and A. V. Kimel, Selective Excitation of Terahertz Magnetic and Electric Dipoles in $\mathrm{Er}^{3+}$ Ions by Femtosecond Laser Pulses in $\mathrm{ErFeO}_{3}$, Phys. Rev. Lett. 118, 017205 (2017). 